\title{
Fracture toughness of titanium-cement interfaces: effects of fibers and loading angles
}

\author{
This article was published in the following Dove Press journal: \\ International Journal of Nanomedicine \\ I April 2014 \\ Number of times this article has been viewed
}

\author{
Morshed Khandaker' \\ Khatri Chhetri Utsaha' \\ Tracy Morris ${ }^{2}$ \\ 'Department of Engineering and \\ Physics, ${ }^{2}$ Department of Mathematics \\ and Statistics, University of Central \\ Oklahoma, Edmond, OK, USA
}

\begin{abstract}
Ideal implant-cement or implant-bone interfaces are required for implant fixation and the filling of tissue defects created by disease. Micron- to nanosize osseointegrated features, such as surface roughness, fibers, porosity, and particles, have been fused with implants for improving the osseointegration of an implant with the host tissue in orthopedics and dentistry. The effects of fibers and loading angles on the interface fracture toughness of implant-cement specimens with and without fibers at the interface are not yet known. Such studies are important for the design of a long-lasting implant for orthopedic applications. The goal of this study was to improve the fracture toughness of an implant-cement interface by deposition of micron- to nanosize fibers on an implant surface. There were two objectives in the study: 1) to evaluate the influence of fibers on the fracture toughness of implant-cement interfaces with and without fibers at the interfaces, and 2) to evaluate the influence of loading angles on implant-cement interfaces with and without fibers at the interfaces. This study used titanium as the implant, poly(methyl methacrylate) (PMMA) as cement, and polycaprolactone (PCL) as fiber materials. An electrospinning unit was fabricated for the deposition of PCL unidirectional fibers on titanium (Ti) plates. The Evex tensile test stage was used to determine the interface fracture toughness $\left(\mathrm{K}_{\mathrm{C}}\right)$ of Ti-PMMA with and without PCL fibers at $0^{\circ}, 45^{\circ}$, and $90^{\circ}$ loading angles, referred to in this article as tension, mixed, and shear tests. The study did not find any significant interaction between fiber and loading angles $(P>0.05)$, although there was a significant difference in the $\mathrm{K}_{\mathrm{C}}$ means of Ti-PMMA samples for the loading angles $(P<0.05)$. The study also found a significant difference in the $\mathrm{K}_{\mathrm{C}}$ means of Ti-PMMA samples with and without fibers $(P<0.05)$. The results showed that the addition of the micron- to nanosize PCL fibers on Ti improved the quality of the Ti-PMMA union. The results of the study are essential for fatigue testing and finite-element analysis of implant-cement interfaces to evaluate the performance of orthopedic and orthodontic implants.
\end{abstract}

Keywords: titanium, cement, interface, PMMA, polycaprolactone, fracture toughness, orthopedics, orthodontics

\section{Introduction}

The fracture toughness of an implant-cement interface is a mechanical property that measures the resistance to propagation of a crack originating at the interface. Our previous research ${ }^{1}$ reported that the addition of micron- and nanosize magnesium oxide particles to poly(methyl methacrylate) (PMMA) improved the fracture toughness of the bone-cement union due to increased surface roughness. ${ }^{2}$ For the same reason, the deposition of the micron- to nanosize polymer fibers to an implant surface can improve the fracture toughness of the implant-cement interface. To the best of the present authors' knowledge, however, no studies of this aspect have been published.
Correspondence: Morshed Khandaker Department of Engineering and Physics, University of Central Oklahoma, 100 North University Drive, Edmond, OK 73034, USA

Tel + I 4059745935

Fax +l 4059743812

Email mkhandaker@uco.edu 
The weakest spots in implant-cement interfaces are the natural flaws along the interface. Such flaws are inevitably introduced during the surgical procedure or from polymerization shrinkage during cement preparation. ${ }^{3}$ Orr et $\mathrm{al}^{4}$ found that cracks observed around hip-prosthesis stems in laboratory specimens of bone cement were due to PMMA cement shrinkage and that residual stresses were sufficient to cause crack initiation prior to functional loading. A comparative study by Kwong and Power ${ }^{5}$ found polymerization shrinkage of orthopedic acrylic bone cements (Palacos ${ }^{\circledR}$ R, Palacos LV [both with gentamicin]; Zimmer, Warsaw, IN, USA; CMW ${ }^{\circledR}$ 1, CMW 2, CMW Endurance, and Simplex; Johnson and Johnson, New Brunswick, NJ, USA). Their studies found shrinkage varied between $3.82 \%$ and 7.08\%, with CMW Endurance having the lowest and Palacos LV the highest. Depending on the orthodontic material, the magnitude of the "free" curing contraction varies from $4 \%$ to $9 \%$ for unfilled resins and from $1 \%$ to $5 \%$ for resin composites and glass polyalkenoate cements. ${ }^{6}$

Among many factors, implant-surface roughness and loading angles at the interface affect the propagation of these flaws, ie, fracture toughness of bone-cement or implant-cement interfaces. ${ }^{7,8} \mathrm{Kim}$ et $\mathrm{al}^{9}$ found that the molecular dissipation of the polymer in the vicinity of the interface was the major cause of the practical energy of separation of polymer-metal interfaces. According to Kim et $\mathrm{al}^{9}$, mechanical interlocks promoted by adsorption provoke energy-dissipation processes during fracture, which practically constitute the adhesion strength of a polymer-metal interface. Microstructural changes at the interface by deposition of fibers on an implant or incorporation of nanoparticles to cement can improve the fracture toughness of implant-cement interfaces. The loading mode controls the mechanical interlock effect, which is attributed to the fact that the stress distribution at the interface controls the deformation and failure characteristics of the polymer resin near the interface. This study estimated the improvement or decrease of the fracture toughness of implantcement interfaces due to the deposition of polymer fibers on titanium (Ti) implants for different loading directions.

Electrospinning is a nanotechnology process by which polymer fibers with micro- to nanometer diameters can be obtained from an electrostatically driven jet of electrostatic polymer solution. ${ }^{10,11}$ These fibers have a high surface areato-volume ratio, which improves the surface properties of biomedical implants. ${ }^{11}$ The improvement of biological and mechanical functions of an implant due to the incorporation of nanofibers has been reported. ${ }^{12}$ However, the interface fracture toughness of the implant-cement interface due to the addition of these fibers has not yet been determined. Several researchers have reported that loading angles have a significant effect on the mechanical strength of bone-cement interfaces. ${ }^{7,13,14}$ The study of the estimation of implantcement interface with and without fibers as a function of different loading angles is important for designing a longlasting implant for orthopedic and orthodontic applications. Two questions will be answered during this study: 1) whether the inclusion of micron- to nanosize polycaprolactone (PCL) fibers has any influence on the fracture toughness of Ti-PMMA interfaces, and 2) whether loading angles have any influence on the fracture toughness of Ti-PMMA interfaces with or without fibers.

\section{Materials and methods \\ Sample preparation}

Ti bars (6Al-4V ELI, ASTM B348 standard, grade 23, biocompatible) of dimensions $22 \times 12 \times 2 \mathrm{~mm}$ were purchased from Titanium Metal Supply, Poway, CA, USA. Cobalt ${ }^{\mathrm{TM}} \mathrm{HV}$ bone cement (Biomet Orthopedics, Warsaw, IN, USA) was used as the PMMA cement. According to manufacturer recommendations, PMMA cement was prepared by hand-mixing $2.2 \mathrm{~g}$ of PMMA powder with $1.1 \mathrm{~mL}$ of methyl methacrylate monomer using a powder: monomer ratio of 2:1. PCL beads and acetone were purchased from Sigma-Aldrich (St Louis, MO, USA). PCL fibers were used because of ease of fiber fabrication, biocompatibility, and cost.

Single-edge crack sandwiched Ti-PMMA specimens without fibers were prepared using the method presented in detail in our earlier study. ${ }^{1}$ Figure 1 schematically represents the process of the fabrication and test configurations (tension, mixed, and shear tests) of single-edge crack sandwiched TiPMMA specimens with fibers. PCL solution was prepared by ultrasonic (Vibra-Cell ${ }^{\mathrm{TM}}$ VCX 130; Sonics and Materials, Newtown, CT, USA) mixing of $7.69 \mathrm{wt} \%$ of PCL pellets (pellet size $\sim 3 \mathrm{~mm}$, average molecular number 80,000 ) with acetone (laboratory reagent $\geq 99.5 \%$ ). Both PCL and acetone were purchased from Sigma-Aldrich. The sonication process was carried out at approximately $80^{\circ} \mathrm{C}$ for an hour.

An electrospinning unit (Figure 2A) was fabricated to deposit aligned unidirectional PCL fibers on Ti plates along the thickness. The solution was poured into a glass syringe in an infusion pump (PHD Ultra ${ }^{\mathrm{TM}}$; Harvard Apparatus, Holliston, MA, USA) for fiber production. PCL fibers were ejected from the glass syringe via charged needle ( $23 \mathrm{G}$ blunt needle, aluminum hub, 1 " length). The needle was charged by a high-voltage power source (ES 30 series; Gamma High Voltage Research, Ormond Beach, FL, USA). The fibers were deposited on a grounded custom-made drum collector. A direct-current motor with the drum was mounted on a precision linear stage (model 426; Newport, Irvine, CA, 


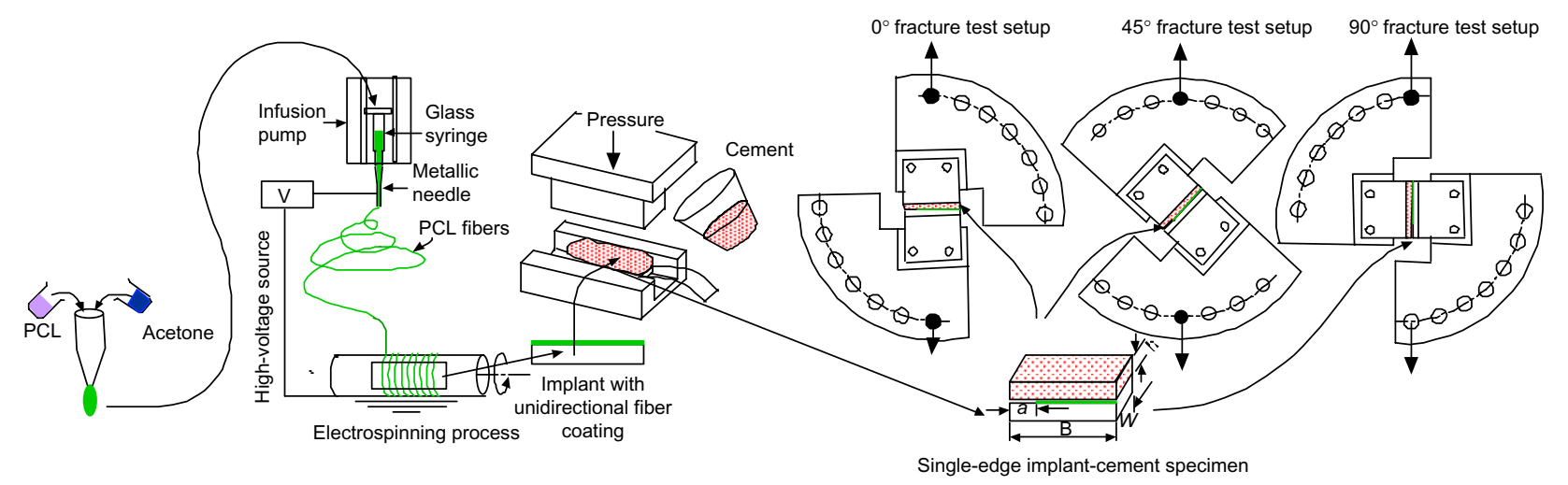

Figure I Schematic representation of the experimental design process used in the study.

Notes: The experiment had four major steps: I) preparation of polycaprolactone (PCL) solution, 2) deposition of unidirectional PCL fibers on a titanium plate, 3) fabrication of Ti-poly (methyl methacrylate) (PMMA) specimens with and without PCL fibers using a custom-made mold that can be found in Khandaker et al' and 4) conducting fracture tests on the sandwiched Ti-PMMA specimens under tension-, mixed-, and shear-load conditions. $\mathbf{a}$ Is the initial crack length, $\mathbf{B}$ is the length, $\mathbf{W}$ is the width and $\mathbf{h}$ shows the thickness of a sample.

USA). The motion of the stage was controlled by a linear actuator (LTA-HS; Newport) to produce aligned unidirectional fibers on the Ti plates. Six Ti plates and a carbon tape were attached on the drum using double-sided tape. Carbon tape was used for the visualization of fiber distribution and measurement of fiber dimension using a Nikon (Tokyo, Japan) SMZ stereomicroscope and a Hitachi (Tokyo, Japan)
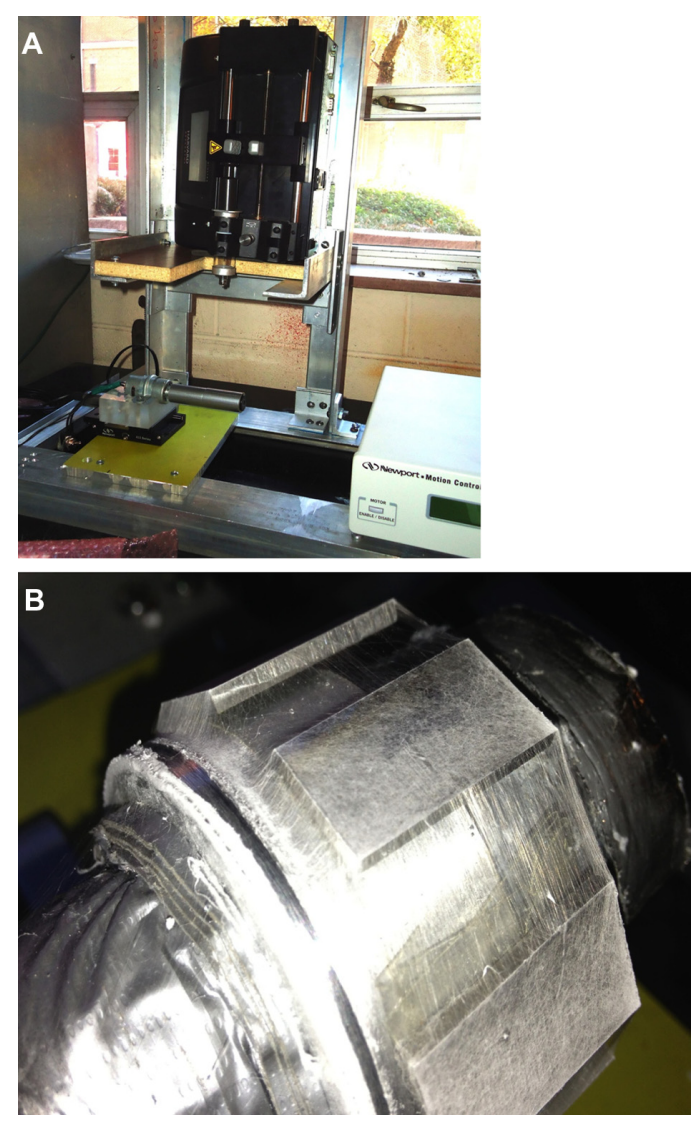

Figure 2 (A) Electrospinning setup used in the study for the fabrication of polycaprolactone fibers; (B) unidirectional fiber deposition on titanium plates.
TM3000 scanning electron microscope (SEM), respectively. The unidirectional fibers were collected on the Ti surface area, as shown in Figure 2B. The method of the creation of single edge crack for Ti-PMMA specimens with fibers was similar as edge crack prepared for Ti-PMMA specimens without fibers. ${ }^{1}$ Nikon SMZ stereomicroscope was used for the measurement of the crack.

\section{Experiments and analysis}

Pullout tests were conducted on the Ti-PMMA samples with and without fibers at room temperature and a loading rate of $0.01 \mathrm{~mm} / \mathrm{second}$ using an Evex tensile stage (Evex Analytical Instruments, Princeton, NJ, USA). Load was applied at $0^{\circ}$, $45^{\circ}$, and $90^{\circ}$ loading angles with respect to the direction of interface in this study. Figure 3 shows the fabricated $0^{\circ}, 45^{\circ}$, and $90^{\circ}$ loading angle test setups for Ti-PMMA specimens using the Evex test stage. The tests were referred to as tension, mixed, and shear tests, respectively. Load and displacement during the fracture tests were continuously recorded until the failure of the Ti-PMMA specimens. Since the critical load ( $\mathrm{P}_{\mathrm{C}}$; load that breaks the interface of the Ti-PMMA samples) depends on the precrack length of the sandwiched samples, the $\mathrm{K}_{\mathrm{C}}$ values of the Ti-PMMA samples were calculated to analyze the experimental results. The $\mathrm{K}_{\mathrm{C}}$ values of the Ti-PMMA samples were calculated according to Wang and Agrawa $~^{13}$ for $0^{\circ}, 45^{\circ}$, and $90^{\circ}$ loading angles, which correspond to tension, mixed, and shear tests, using:

$$
K_{C}=\frac{P_{C} \lambda^{\psi} \sqrt{\pi a}}{B W} \sqrt{\left(A_{I} \cos \theta\right)^{2}+\left(A_{I I} \sin \theta\right)^{2}}
$$

where $\theta$ is the loading angles, and $\lambda$ is a scale factor determined using:

$$
\lambda=\sqrt{\frac{1-\alpha}{1-\beta^{2}}}
$$




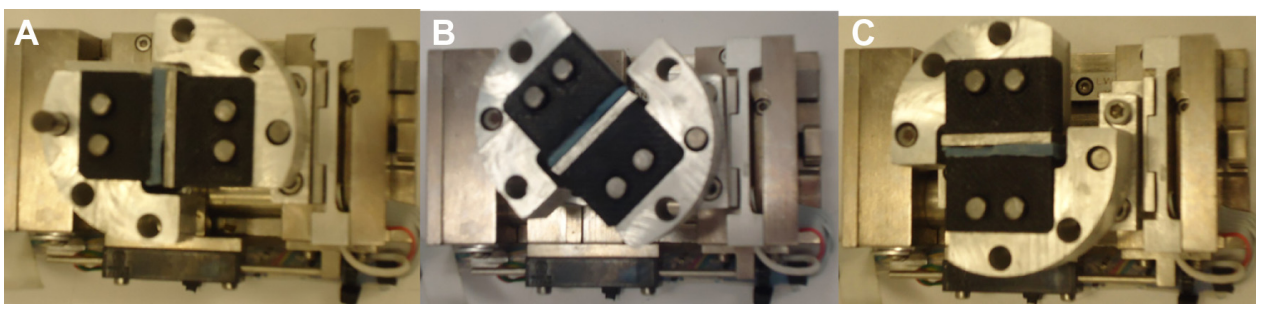

Figure 3 Fabricated experimental setup for (A) tension, (B) mixed, and (C) shear tests on titanium-poly(methyl methacrylate) samples using the Evex (Evex Analytical Instruments, Princeton, NJ, USA) mechanical test stage.

The values of $\alpha$ and $\beta$ are Dundurs parameters, ${ }^{15}$ which estimate the elastic mismatch across the bimaterial interface, given by:

$$
\begin{aligned}
& \alpha=\frac{E_{1}\left(1-v_{2}^{2}\right)-E_{2}\left(1-v_{1}^{2}\right)}{E_{1}\left(1-v_{2}^{2}\right)+E_{2}\left(1-v_{1}^{2}\right)} \\
& \beta=\frac{E_{1}\left(1-v_{2}-2 v_{2}^{2}\right)-E_{2}\left(1-v_{1}-2 v_{1}^{2}\right)}{2 E_{1}\left(1-v_{2}^{2}\right)+2 E_{2}\left(1-v_{1}^{2}\right)},
\end{aligned}
$$

where $E_{1}, E_{2}$, and $v_{1}$, and $v_{2}$ are elastic moduli and Poisson's ratios of the cement and titanium, respectively. The values of $E$ and $v$ for PMMA were considered as $740.41 \mathrm{MPa}$ and 0.184 , respectively. ${ }^{1}$ The values of $E$ and $v$ for Ti were considered as $110 \mathrm{GPa}$ and 0.342 , respectively (Titanium Metal Supply, Inc., email communication, June, 2013). In equation $1, \psi$ is a correction factor, which accounts for the interlayer thickness effect, determined using: ${ }^{13}$

$$
\psi=e^{-p(h / W)^{k}},
$$

where $h$ is the interlayer thickness, as shown in Figure 1. In equation $4, p$ and $k$ are functions of loading angle, $\theta$, and are given as:

$$
\begin{aligned}
& p=5.056-1.20 \theta+266 \theta^{2} \\
& k=0.777-0.124 \theta-0.028 \theta^{2},
\end{aligned}
$$

where $\theta$ is in radians. In equation $1, A_{\mathrm{I}}$ and $A_{\mathrm{II}}$ are shape functions determined using: ${ }^{13}$

$$
\begin{aligned}
& A_{I}=\frac{1}{1-\rho} \sqrt{\frac{0.26+2.65 \frac{\rho}{1-\rho}}{1+0.55 \frac{\rho}{1-\rho}-0.08\left(\frac{\rho}{1-\rho}\right)^{2}}} \\
& A_{I I}=\frac{1}{1-\rho} \sqrt{\frac{-0.23+1.40 \frac{\rho}{1-\rho}}{1-0.67 \frac{\rho}{1-\rho}+2.08\left(\frac{\rho}{1-\rho}\right)^{2}}},
\end{aligned}
$$

where $\rho=a / W$. Initial crack length, thickness, and width of a specimen are given by $a, B$, and $W$, respectively. It is important to mention that the PCL fiber layer between the Ti and PMMA was ignored in the analysis, because it was extremely thin compared to the thickness of the Ti and PMMA.

\section{Statistical analysis}

A two-factor analysis of variance was performed, followed by multiple comparisons with Tukey's adjustment. The two factors were loading angle (tension, mixed, or shear) and fibers (with or without). Results were considered significant at $P<0.05$. All analysis was performed on SAS version 9.1 (SAS Institute, Cary, NC, USA).

\section{Results and discussion}

PCL fibers were successfully produced using the electrospinning unit. The produced fibers on carbon tape were visualized and fiber-coating thickness measured using the Nikon stereomicroscope (Figure 4A). The topography of the fibers on carbon tape was analyzed using the Hitachi SEM (Figure 4B-D). It is clear from the stereomicroscope and SEM images that the produced fiber is unidirectional, although overlapping of the fibers along the direction was observed. The thickness of fiber coating on the carbon tape was found to be in the range of 5.85-8.97 $\mu \mathrm{m}$. Table 1 reports the topographical information of fiber distribution for three arbitrarily selected carbon-tape samples (Figure 4B-D). Since the fibers were collected on the carbon tape and Ti plates at the same time in the drum collector, the diameter of PCL fibers on Ti plates must also be in the micron range $(0.98-6.29 \mu \mathrm{m})$. Table 1 shows a $20 \mu \mathrm{m}$ line intersected 6-9 fibers perpendicular to the direction of the fiber, and the maximum distance between two fibers was $49.71 \mu \mathrm{m}$. Figure 5 shows the load-displacement curves of Ti-PMMA specimens with and without fibers under tension, mixed, and shear loadings. The load-displacement responses of all different kinds of Ti-PMMA specimens are characterized by elastic response and then sudden failure of the specimen without a noticeable plastic region. This means 


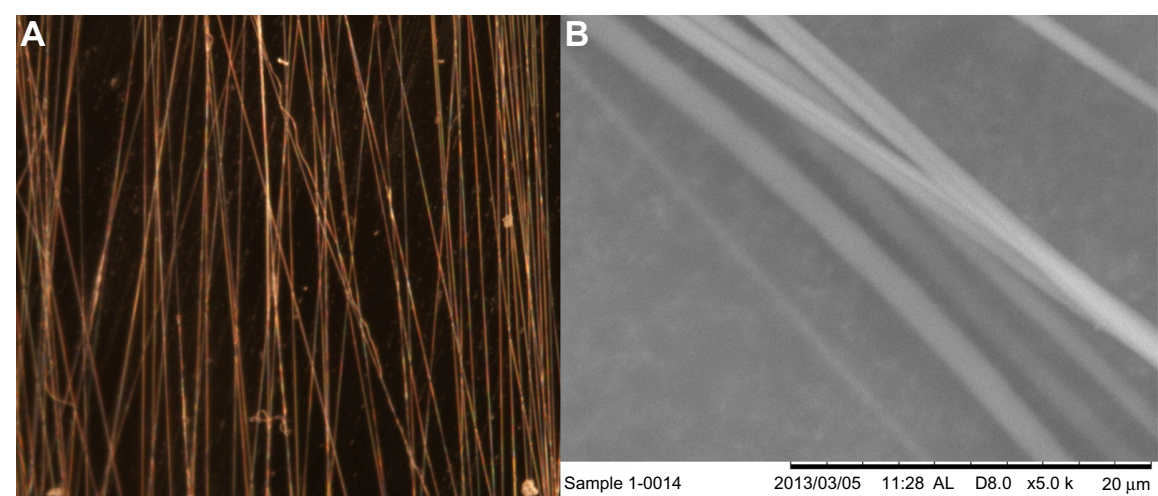

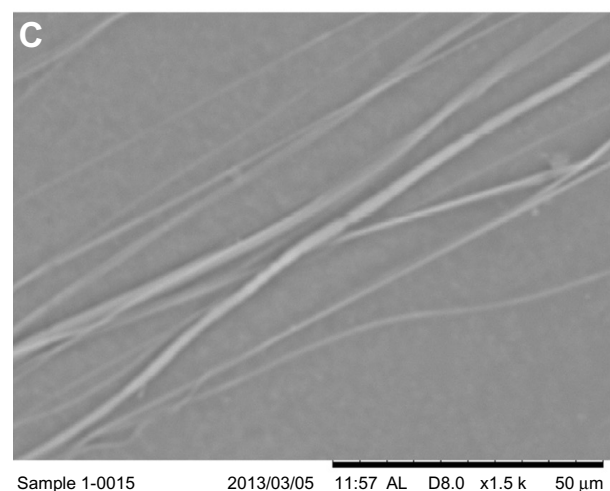

Figure 4 (A) Stereomicroscope image of unidirectional fibers at $8 \times$ magnification,

interfacial softening was not found by the addition of fibers at the Ti-PMMA interface. Figure 5 shows a clear difference of critical fracture load between Ti-PMMA specimens with and without fibers under tension, mixed, and shear loadings. Table 2 presents the descriptive statistics of the dimension of different groups of Ti-PMMA specimens. Table 2 shows that the edge cracks prepared for Ti-PMMA specimens with and without fibers were in the range of 7-10 $\mathrm{mm}$.

There was no significant interaction between loading angle and fiber $\left(F_{[2,12]}=0.88, P=0.4404\right)$ from the calculated $\mathrm{K}_{\mathrm{C}}$ of different groups of Ti-PMMA specimens. No interaction means that for each loading angle, the same relation-

Table I Topographical information of fiber distribution on carbon tape

\begin{tabular}{llll}
\hline Description & \multicolumn{3}{l}{ Sample number } \\
\cline { 2 - 4 } & $\mathbf{I}$ & $\mathbf{2}$ & $\mathbf{3}$ \\
\hline $\begin{array}{l}\text { Number of fibers } \\
\text { intersecting }(20 \mu \mathrm{m}-\end{array}$ & 7 & 9 & 6 \\
$\begin{array}{l}\text { length line) } \\
\begin{array}{l}\text { Ranges of diameter } \\
\text { of fibers }\end{array}\end{array}$ & $1.22-6.29 \mu \mathrm{m}$ & $0.98-2.32 \mu \mathrm{m}$ & $1.33-3.28 \mu \mathrm{m}$ \\
$\begin{array}{l}\text { Ranges of distance } \\
\text { between two fibers }\end{array}$ & $1.18-46.45 \mu \mathrm{m}$ & $4.4 \mathrm{I}-18.5 \mu \mathrm{m}$ & $2.5-49.7 \mathrm{I} \mu \mathrm{m}$ \\
\hline
\end{tabular}

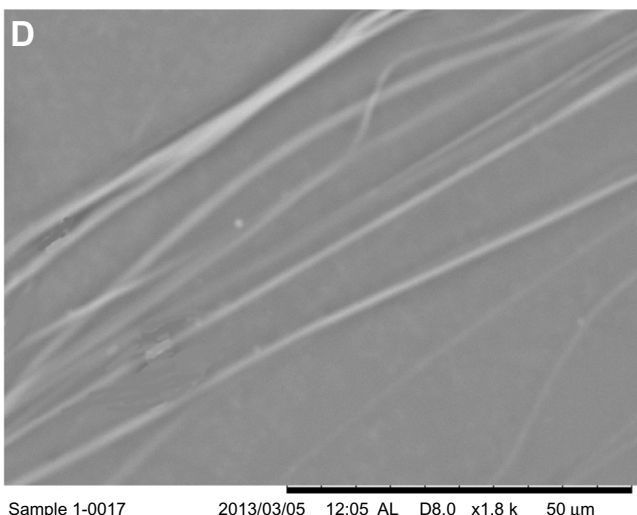

Sample 1-0017

-D) scanning electron microscopy images of three samples.

ship of $\mathrm{K}_{\mathrm{C}}$ existed between the with-fiber and without-fiber samples. There was, however, a significant difference in the mean fracture toughness for the loading angles $\left(F_{[2,12]}=18.27\right.$, $P<0.001)$ and fibers $\left(F_{[1,12]}=32.08, P<0.001\right)$ separately. Specifically, the mean fracture toughness was significantly lower for mixed than for tension or shear loads. Also, the mean fracture toughness was significantly lower without fibers than with fibers. The experimental results revealed that the mean $\mathrm{K}_{\mathrm{C}}$ value of the Ti-PMMA samples with fibers was higher compared to the mean $\mathrm{K}_{\mathrm{C}}$ value of Ti-PMMA samples without fibers for all loading angles. These results are displayed in Figures 6 and 7. Since the interaction between loading angle and fibers was not significant at the 0.05 level, the test for differences in loading angles was performed by combining the with- and without-fiber samples (Figure 6). Similarly, a test for differences in the with- and without-fiber samples was performed by combining the tension, mixed, and shear samples (Figure 7). Therefore, Figure 7 displays the mean interface fracture toughness of the tension, mixed, and shear samples combined.

This observed difference of the $\mathrm{K}_{\mathrm{C}}$ values between Ti-PMMA samples with and without fibers is due to the differences in surface roughness at the interfaces of 


\section{A}
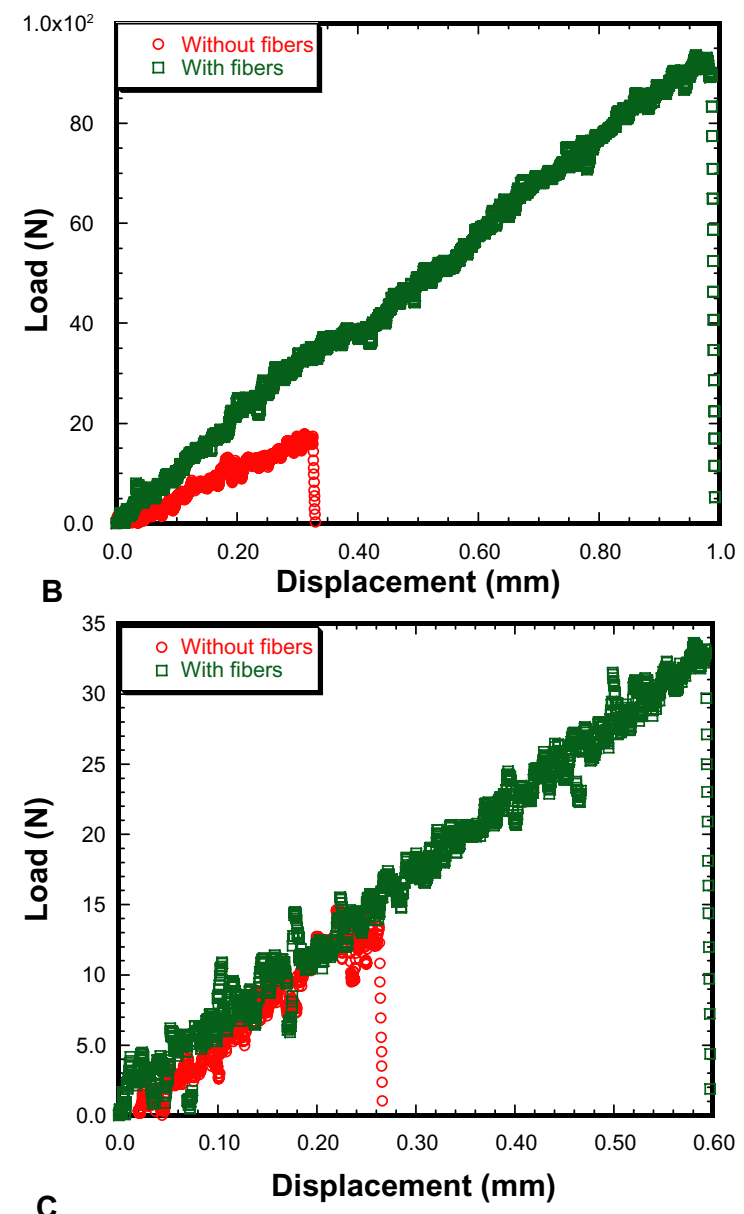

C

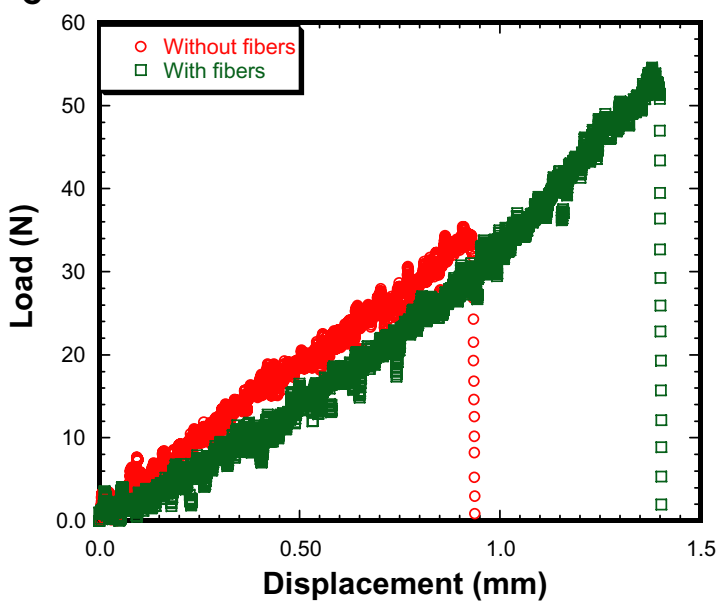

Figure $\mathbf{5}$ Load versus displacement graphs of titanium-poly(methyl methacrylate) samples under (A) tension, (B) mixed, and (C) shear load.

Ti-PMMA with fibers compared to those Ti-PMMA samples without fibers. An increase of surface roughness led to a decrease of micromovement of cracks at the interface of Ti-PMMA samples with fibers, thus increasing the load- and displacement-bearing capacity of Ti-PMMA samples with, compared to Ti-PMMA samples without, fibers (Figure 5).
This behavior is consistent with published research, ${ }^{8,14,16-18}$ where it was found that increased surface roughness helped strengthen the interfacial mechanical properties of bonecement or implant-cement interfaces. The observed loading effect on Ti-PMMA specimens can be explained by the fact that the elastic-plastic behavior and crack-tip stress concentration at the interface impacted on the $\mathrm{K}_{\mathrm{C}}$ of the specimens due to the variation of the loading angles. ${ }^{9,13}$ The observed nonsignificant interaction between fiber and loading angles $(P=0.4404)$ can be explained by the fact that the addition of the fibers at the Ti-PMMA interface pacified the impact of the correction factor $\psi$ and bimaterial stress-intensity factors $\left(A_{\mathrm{I}} \cos \theta, A_{\mathrm{II}} \sin \theta\right)$ on the $\mathrm{K}_{\mathrm{C}}$ due to the variation of loading angles. The mechanism of such pacification on the $\mathrm{K}_{\mathrm{C}}$ of sandwiched specimens by the addition of fibers at the interface should be further investigated.

There are no publications on the $\mathrm{K}_{\mathrm{C}}$ of Ti-PMMA specimens with and without fibers or loading angle effects on such specimens with which to compare our results. However, the trend of the $\mathrm{K}_{\mathrm{C}}$ of Ti-PMMA interface without fiber as a function of the loading angles is in agreement with Wang and Agrawal, ${ }^{13}$ but contradicts Mann et al ${ }^{19}$ and Wang et al. ${ }^{7}$ According to Wang and Agrawal, the $\mathrm{K}_{\mathrm{C}}$ of a bone-cement interface decreased as loading angle increased, approached its lowest value at $60^{\circ}(P<0.05)$, and increased to a maximum value at $90^{\circ}$. The present study selected $0^{\circ}, 45^{\circ}$, and $90^{\circ}$ loading angles due to the fact that they produce pure normal, combined (due to both normal and shear stress) and pure shear stresses along the direction of the interface. Since the goal of this study was to evaluate the effect of these stresses on the $\mathrm{K}_{\mathrm{C}}$ of Ti-PMMA interfaces, this study did not find the angle at which the value of $\mathrm{K}_{\mathrm{C}}$ of Ti-PMMA is lowest. According to Mann et al and Wang et al, there was a significant increase in mechanical strength $(P<0.001)$ of sandwiched bone-cement specimens with increasing mixedmode loading angles. This contradiction could be justified by the fact that this study used a fracture-mechanics technique (based on precracked sandwiched specimens) instead of a nominal strength-measurement technique (based on defect-free sandwiched specimens). The fracture-mechanics technique was used in this study as it provides more fundamental and meaningful estimation of the $\mathrm{K}_{\mathrm{C}}$ of sandwiched specimens than the nominal strength-measurement technique used by previous authors. ${ }^{7,19}$

The formulas used for calculating the $\mathrm{K}_{\mathrm{C}}$ for Ti-PMMA specimens take into account the influence of material combination by scale factor, $\lambda$ and finite interlayer thickness, $h$ by correction factor $\psi$. The material properties (elastic 
Table 2 Descriptive statistics (means \pm standard deviation) of the experimental single-edge sandwiched titanium-poly(methyl methacrylate) specimens

\begin{tabular}{|c|c|c|c|c|c|c|}
\hline \multirow[t]{2}{*}{ Description } & \multicolumn{2}{|l|}{ Tension } & \multicolumn{2}{|l|}{ Mixed } & \multicolumn{2}{|l|}{ Shear } \\
\hline & Without fiber & With fiber & Without fiber & With fiber & Without fiber & With fiber \\
\hline Number of specimens & 3 & 3 & 3 & 3 & 3 & 3 \\
\hline Average width, W (mm) & $23.15 \pm 0.30$ & $21.7 \pm 1.99$ & $23.06 \pm 0.27$ & $23.13 \pm 0.39$ & $22.76 \pm 0.4 I$ & $22.75 \pm 0.37$ \\
\hline Average thickness, $B(\mathrm{~mm})$ & $12.15 \pm 0.11$ & $11.17 \pm 1.06$ & $12.11 \pm 0.03$ & $12.16 \pm 0.07$ & $12.1 \pm 0.03$ & $\mid 1.93 \pm 0.31$ \\
\hline Average crack length, a (mm) & $10.08 \pm 0.33$ & $7.26 \pm 1.67$ & $10.44 \pm 0.40$ & $9.79 \pm 0.40$ & $10.22 \pm 0.28$ & $9.95 \pm 0.26$ \\
\hline Interface fracture toughness, $\mathrm{K}_{\mathrm{C}}\left(\mathrm{KPa} \cdot \mathrm{m}^{1 / 2}\right)$ & $11.90 \pm 1.09$ & $20.98 \pm 2.47$ & $6.15 \pm 1.53$ & $12.25 \pm 3.06$ & $|4.7| \pm 1.03$ & $20.09 \pm 1.26$ \\
\hline
\end{tabular}

moduli and Poisson's ratios) of PCL and the thickness of the PCL layer was ignored for the determination of scale, and correction factors assuming their effects on the factors are negligible. Principles of interfacial fracture mechanics for two materials and the linear finite element analysis (FEA) method were used for the determination of scale and correction factors, respectfully. Further studies are required to develop a theoretical model and nonlinear finite element model that represent fiber-induced implant/biomaterial systems to calculate accurately the values of scale and correction factors for the determination of $\mathrm{K}_{C}$.

A limitation of the study was the variability of amount of fiber deposition and diameter of the fibers. Fibers were deposited on Ti plates (average $0.002 \mathrm{~g}$ ) for 30 seconds to reduce the variability in fiber amounts. It was found that stiffness, size, and distribution of fibers on Ti plates depends upon the PCL solution viscosity, direct-current voltage, solution flow rate, and time gap between fiber deposition on Ti plates and fracture tests on the Ti-PMMA samples. The authors were careful to minimize the variability of these parameters during experiments. This study was limited to the estimation of the fracture toughness of implant-cement interfaces due to the deposition of polymer fibers on Ti implant for different loading directions. The estimation of the improvement or decrease of the fracture toughness of implant-cement interfaces due to the deposition of nanoparticle additives mixed with cement on Ti implant (with and without PCL fibers) is not known and currently under investigation.

This study advanced interface tissue-engineering research by providing an understanding of how the fracture toughness of tissue-implant or implant-implant interfaces could be improved by surface modification of implant material by micron- to nanosize fibers. The results of the study can be used for fatigue testing and FEA of the implant-cement interfaces, with and without fibers, to evaluate the effect of fibers on the performance of orthopedic and orthodontic implants. For example, the authors are currently conducting pullout fatigue tests on round fiber-coated Ti-PMMA and implant-shape fiber-coated TiPMMA models that resemble the clinical orthodontic and

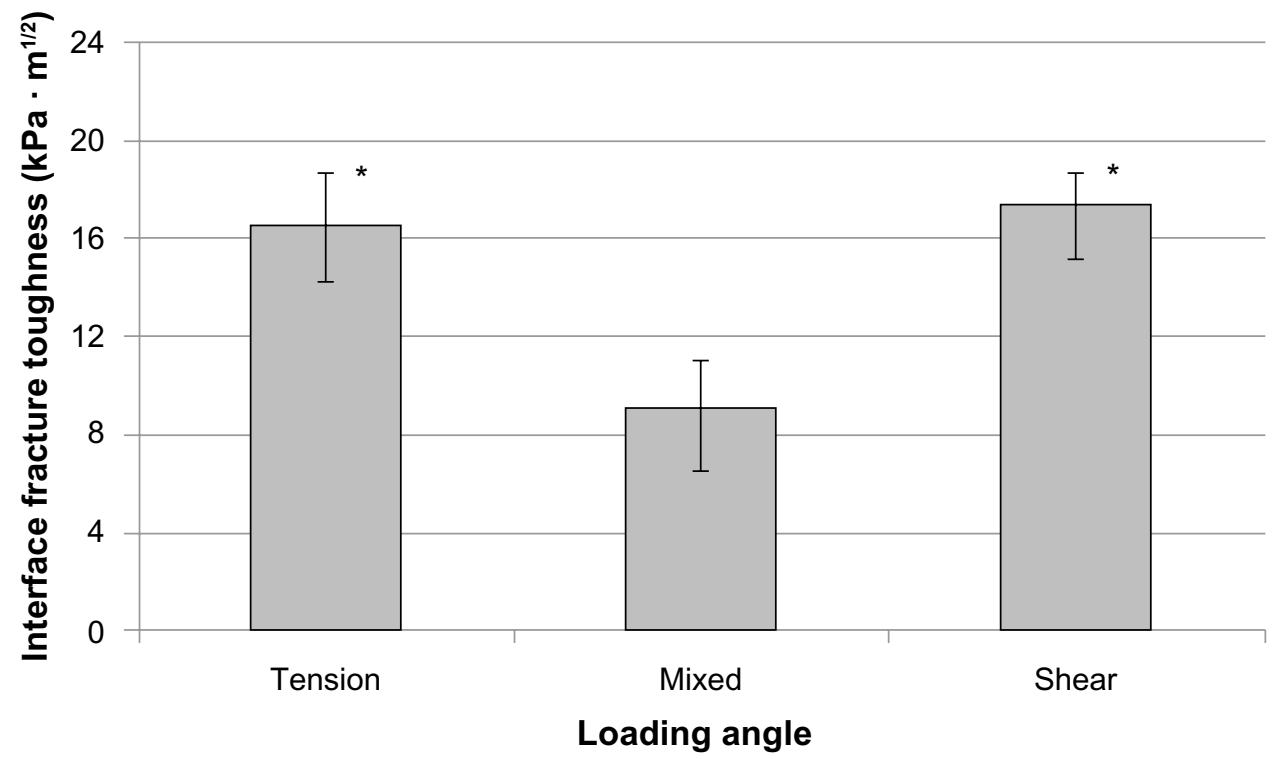

Figure 6 Fracture toughness of the titanium-poly(methyl methacrylate) interface as a function of loading angle. Data presented as means \pm standard error of mean; $\mathrm{n}=6$. Note: $* P<0.01$ (compared to mixed). 


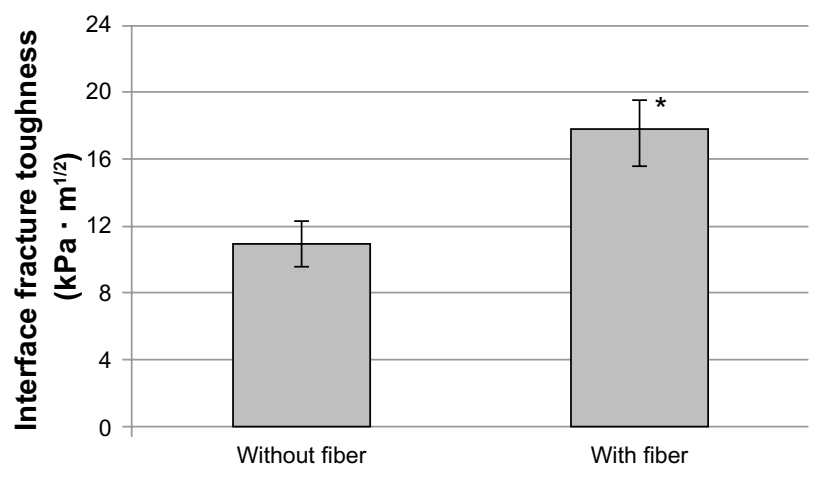

Figure 7 Fracture toughness of the titanium-poly(methyl methacrylate) interface as a function of fiber application. Data presented as means \pm standard error of mean; $n=9$. Note: $* P<0.001$ (compared to without fiber).

orthopedic implant models, respectively. The fracture loads for Ti-PMMA samples with fibers and without fibers at the interface under shear load, obtained from the present study, have been used to estimate the preload for the fatigue tests. Furthermore, the contact stiffness of Ti-PMMA specimens with and without fibers under shear load found in the present study have been used to develop an FEA model of the implant-cement bond under physiological cyclic loading. The FEA model evaluates the effect of fiber coating on the fatigue life of Ti-PMMA interfaces. The results of previous studies will appear in different journals. The experimental setup developed in this study can also be applied for in vitro and in vivo studies to measure fiber-coating effects on implant-cement or cement-bone interfaces. The novelty of this study is the in vitro estimation of fracture toughness of the implant-cement interface with and without micron- to nanosize fibers as a function of loading angles. Further improvement of the interface fracture toughness of Ti-PMMA is possible by depositing nanosize, and more adhesive, polymeric fibers on Ti as well as the inclusion of nanoparticles with PMMA cement.

\section{Conclusion}

This study evaluated the fracture toughness of titaniumcement interfaces with and without unidirectional fibers under $0^{\circ}$ (tension), $45^{\circ}$ (mixed), and $90^{\circ}$ (shear) loading angles. This study found the following:

1. no significant interaction of fracture toughness of Ti-PMMA data between fibers and loading angles $(P>0.05)$;

2. the mean value of fracture toughness of Ti-PMMA samples with fibers was higher compared to those Ti-PMMA samples without fibers for all loading angles $(P<0.05)$;
3. loading has a significant effect on the fracture toughness of Ti-PMMA samples $(P<0.05)$.

The significance of this result is that load-invariant implant-cement interfaces can be created by the application of fibers at the interface. This finding suggests that integration of fibers with Ti should be further investigated with respect to applications in total joint arthroplasty and orthodontic implants.

\section{Acknowledgments}

This research was made possible by grant 8P20GM103447 from the US National Institutes of Health and an on-campus faculty grant program from the University of Central Oklahoma Office of Research and Grants. Special thanks to Dr Timothy W Teske, DO, Orthopedic Surgery, Enid, OK for demonstrating the PMMA cement-preparation protocols.

\section{Disclosure}

The authors report no conflicts of interest in this work.

\section{References}

1. Khandaker M, Li Y, Morris T. Micro and nano MgO particles for the improvement of fracture toughness of bone-cement interfaces. J Biomech. 2013;46(5):1035-1039.

2. Ricker A, Liu-Snyder P, Webster TJ. The influence of nano $\mathrm{MgO}$ and $\mathrm{BaSO}_{4}$ particle size additives on properties of PMMA bone cement. Int J Nanomedicine. 2008;3(1):125-132.

3. McCormack BA, Prendergast PJ. Analysis of crack propagation paths at implant/bone-cement interfaces. J Biomech Eng. 1996;118(4): $579-585$.

4. Orr JF, Dunne NJ, Quinn JC. Shrinkage stresses in bone cement. Biomaterials. 2003;24(17):2933-2940.

5. Kwong FN, Power RA. A comparison of the shrinkage of commercial bone cements when mixed under vacuum. J Bone Joint Surg Br. 2006;88(1):120-122.

6. Davidson CL, Feilzer AJ. Polymerization shrinkage and polymerization shrinkage stress in polymer-based restoratives. $J$ Dent. 1997;25(6):435-440.

7. Wang JY, Tozzi G, Chen J, Contal F, Lupton C, Tong J. Bone-cement interfacial behaviour under mixed mode loading conditions. $J$ Mech Behav Biomed Mater. 2010;3(5):392-398.

8. Walsh WR, Svehla MJ, Russell J, et al. Cemented fixation with PMMA or Bis-GMA resin hydroxyapatite cement: effect of implant surface roughness. Biomaterials. 2004;25(20):4929-4934.

9. Kim WS, Yun IH, Lee JJ, Jung HT. Evaluation of mechanical interlock effect on adhesion strength of polymer-metal interface. Int JAdh Adhes. 2010;30(6):408-417.

10. Wang HB, Mullins ME, Cregg JM, et al. Creation of highly aligned electrospun poly-L-lactic acid fibers for nerve regeneration applications. J Neural Eng. 2009;6(1):016001.

11. Kwon IK, Kidoaki S, Matsuda T. Electrospun nano- to microfiber fabrics made of biodegradable copolyesters: structural characteristics, mechanical properties and cell adhesion potential. Biomaterials. 2005;26(18):3929-3939.

12. Nair L, Laurencin C. Nanofibers and nanoparticles for orthopaedic surgery applications. J Bone Joint Surg Am. 2008;90 Suppl 1:128-131.

13. Wang XD, Agrawal CM. A mixed mode fracture toughness test of bonebiomaterial interfaces. J Biomed Mater Res. 2000;53(6):664-672. 
14. Zelle J, Janssen D, Peeters S, Brouwer C, Verdonschot N. Mixed-mode failure strength of implant-cement interface specimens with varying surface roughness. J Biomech. 2011;44(4):780-783.

15. Dundurs J. Discussion: "Edge-bonded dissimilar orthogonal elastic wedges under normal and shear loading" (Bogy, DB, 1968, ASME J Appl Mech, 35, pp. 460-466). J Appl Mech. 1969;36(3): $650-652$.

16. Ramaniraka N, Rakotomanana L, Leyvraz P. The fixation of the cemented femoral component. Effects of stem stiffness, cement thickness and roughness of the cement-bone surface. J Bone Joint Surg Br. 2000;82(2):297-303.
17. Wang CX, Tong J. Interfacial strength of novel PMMA/HA/nanoclay bone cement. Biomed Mater Eng. 2008;18(6):367-375.

18. Khandaker M, Tarantini S. Material mismatch effect on the fracture of a bone-composite cement interface. Adv Mater Sci Appl. 2012; 1(1):1-8.

19. Mann KA, Mocarski R, Damron LA, Allen MJ, Ayers DC. Mixed-mode failure response of the cement-bone interface. J Orthop Res. 2001;19(6): 1153-1161.
International Journal of Nanomedicine

\section{Publish your work in this journal}

The International Journal of Nanomedicine is an international, peerreviewed journal focusing on the application of nanotechnology in diagnostics, therapeutics, and drug delivery systems throughout the biomedical field. This journal is indexed on PubMed Central,

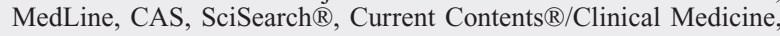

\section{Dovepress}

Journal Citation Reports/Science Edition, EMBase, Scopus and the Elsevier Bibliographic databases. The manuscript management system is completely online and includes a very quick and fair peer-review system, which is all easy to use. Visit http://www.dovepress.com/ testimonials.php to read real quotes from published authors.

Submit your manuscript here: http://www.dovepress.com/international-journal-of-nanomedicine-journal 